See discussions, stats, and author profiles for this publication at: https://www.researchgate.net/publication/235777073

\title{
Diffraction of an optical pulse as an expansion in ultrashort orthogonal Gaussian beam modes
}

Article in Journal of the Optical Society of America A · February 2013

DOI: 10.1364/JOSAA.30.000215 · Source: PubMed

CITATIONS

8

2 authors:

Ronan Mahon

15 PUBLICATIONS 152 CITATIONS

SEE PROFILE

Some of the authors of this publication are also working on these related projects:

Herschel-HIFI View project

452

John Anthony Murphy

National University of Ireland, Maynooth

379 PUBLICATIONS 18,815 CITATIONS

SEE PROFILE 


\title{
Diffraction of an optical pulse as an expansion in ultrashort orthogonal Gaussian beam modes
}

\author{
Ronan J. Mahon* and J. Anthony Murphy \\ Department of Experimental Physics, National University of Ireland, Maynooth, Co. Kildare, Ireland \\ ${ }^{*}$ Corresponding author: ronanjmahon@gmail.com
}

Received August 17, 2012; revised December 6, 2012; accepted December 9, 2012; posted December 12, 2012 (Doc. ID 174497); published January 14, 2013

\begin{abstract}
The Laguerre-Gaussian (LG) beam expansion is described as a numerical and physical model of paraxial ultrashort pulse diffraction in the time domain. An overview of the dynamics of higher-order ultrashort planar LG modes is given through numerical simulations, and the finite width of these beams is shown to induce a dispersive-like axial broadening of the fields, which creates related variations in the on-axis amplitude of such pulses. The propagation of a pulsed plane wave scattered at an aperture is then illustrated as a finite weighted sum of individual planar LG pulses, which allows for intuitive illustration of the convergence of this expansion technique. By applying such an expansion to diffraction at a hard aperture, the planar pulsed LG beams are described as the paraxial analogs of the Bessel boundary waves typically observed in such situations, with both exhibiting superluminal group velocities along the optical axis. Numerical results of pulse diffraction at an aperture highlight the suitability of the LG expansion method for efficient and practical simulation of ultrashort fields in the paraxial regime. (c) 2013 Optical Society of America

OCIS codes: $\quad 320.5550,050.1940,050.5080,260.1960,260.2030$.
\end{abstract}

\section{INTRODUCTION}

While the study of ultrashort pulse dynamics is a comparatively recent addition to the field of optics, massive experimental advancements have been made in the last decade or so, with electromagnetic fields now being generated with durations on attosecond timescales [1,2]. In applied optics, the numerical techniques used to predict the temporal behavior of ultrashort signals are naturally derived from the models of continuous wave $(\mathrm{CW})$ propagation, and methods vary from computationally intensive full-wave solutions of Maxwell's equations such as the finite-difference time-domain (FDTD) algorithm [3-6] to, with more relevance to large optical systems, the diffraction integral approach [7-13]. To introduce in this paper the dynamics involved in pulse diffraction, a generic illustration of the near-field propagation of an ultrashort plane wave pulse truncated at a narrow aperture is shown in Fig. 1.

In this paper we describe a pulse diffracted at an aperture as a superposition of a multitude of component LaguerreGaussian (LG) "pulselets" and relate the propagation of such fields to the observed features of the total diffracted field. In particular, we give a description of how the propagation of these LG modes relates to the fundamental description of pulse diffraction presented by Horváth and co-workers in $[14,15]$. Those works described the diffraction of a short pulse at an aperture in terms of two separate wave components. With the incidence of a plane wave pulse upon an aperture, as in Fig. 1, a GW propagates parallel to the optical axis with the same transverse extent as the aperture, while BWs originate as spherical wavelets at the aperture edge. These two forms of waves can be considered to propagate independently of each other but interfere to form the total observed field. Although this concept of diffraction dates back to the work of Thomas Young in the 19th Century, Horváth et al.'s seminal application of this theory in the modern analysis of pulses allows for a physically intuitive description of the propagation of such fields and is of current interest, with the theory being applied as an accurate and efficient method for the simulation of optical pulse diffraction [16-20]. The physical appearance of the BWs in ultrashort pulse diffraction has been described as being analogous to the Poisson/Arago spot, exhibited recently with high-resolution experimental measurements of femtosecond fields in the time domain using interferometric methods [16-18].

For our description of pulse diffraction at an aperture, the obvious structural similarity of the LG and Bessel functions is addressed. The formation of the observed BWs, with similar propagation characteristics to the Bessel X-waves [16,21], is described in the paraxial regime through the application of an LG expansion in which the expansion coefficients of an aperture function imply the propagation of specific individual planar LG pulses as the paraxial analogs of the BWs, which to our knowledge is an original interpretation. This analysis connects in a simple manner the propagation features of such BWs with the diffraction characteristics of planar Gaussian pulses, illustrating the generality of superluminal axial velocities in the propagation of fields with finite sources, as suggested in [22].

From numerical simulations of the propagation of planar LG pulses with large indices we identify a variation of the on-axis temporal duration of such pulses, which we attribute to the influence of the Gouy phase shift as an analogous freespace refractive index. In reference to this explanation, the group velocity effects identified in a previous work represent the first-order influence of a free-space Gouy dispersion [22], while the features of axial broadening illustrated here are related to second-order characteristics, conventionally associated with the "stretching" of pulses observed in dispersive media. 

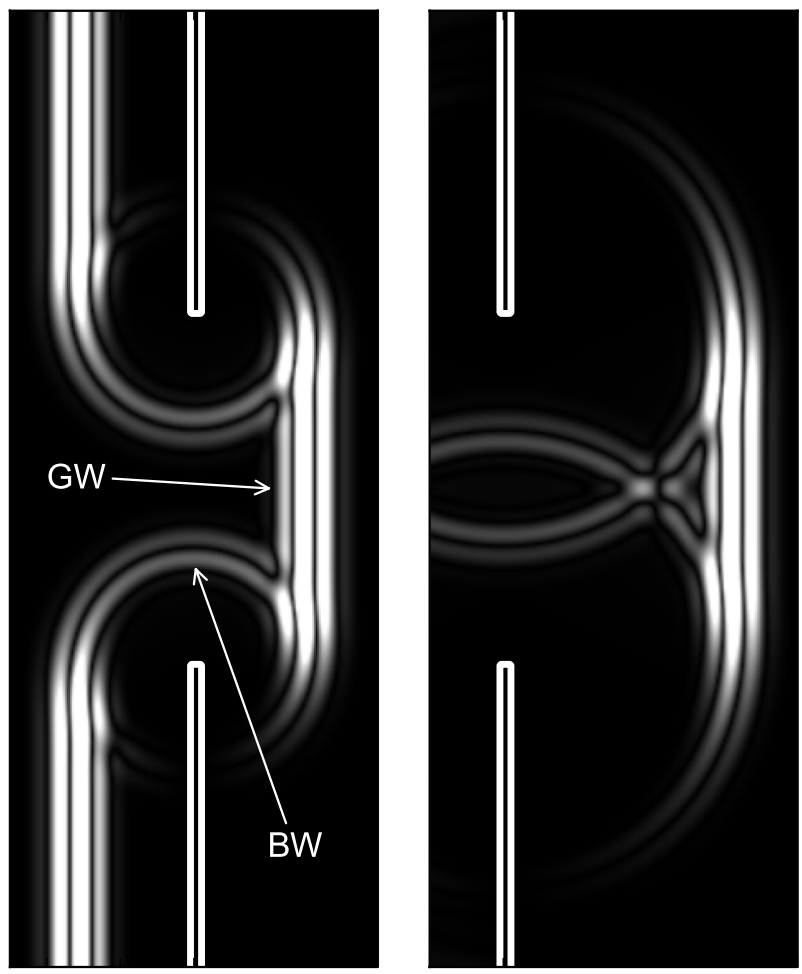

Fig. 1. FDTD simulation of the general behavior of a 1D ultrashort plane wave pulse, propagating from left to right, scattered at a narrow aperture. The propagation of the generated boundary waves (BWs) and geometric wave (GW) is indicated. The field is shown as the absolute value of the electric field and clipped to enhance the BWs.

\section{LAGUERRE-GAUSSIAN FINITE SERIES EXPANSION}

A standard scheme by which the diffraction of a pulse is calculated is through transformation of a field's amplitude excitation signal $s(t)$ from the time domain into a Fourier representation in angular frequency space $S(\omega)$. The diffracted fields of each frequency component $\omega$ can be calculated separately and then integrated with weighting $S(\omega)$ over all frequencies to derive the total scalar electric field of the pulse through the inverse Fourier transform, i.e.,

$$
E(r, z, t)=\frac{1}{\sqrt{2 \pi}} \int_{-\infty}^{\infty} S(\omega) E_{\mathrm{CW}}(\omega, r, z) \exp (-i \omega t) \mathrm{d} \omega,
$$

where $E_{\mathrm{CW}}$ can represent a diffracted CW field with angular frequency $\omega$, generally regardless of the diffraction method applied, be it analytical or numerical, paraxial or complete. Here, we apply the very well known but, in general, rarely applied technique for the computation of paraxial diffraction effects given by the orthogonal LG beam expansion [23-29].

The symmetrical LG functions are given in normalized form as

$$
\psi_{m}\left(r_{0}\right)=\sqrt{\frac{2}{\pi w_{0}^{2}}} L_{m}\left(2 \frac{r_{0}^{2}}{w_{0}^{2}}\right) \exp \left(-\frac{r_{0}^{2}}{w_{0}^{2}}\right)
$$

where $r_{0}$ is the radial coordinate at a reference plane (aperture), $L_{m}$ is the Laguerre polynomial of index $m$, the real positive number $w_{0}$ is the width parameter, and the square root term is a normalization factor. The value of $w_{0}$ as an argument of the Laguerre polynomial also influences the periodicity of the modes, but this maintains the same relation to the overall width of the function. The application of these functions in optics is based on their existence as solutions to the cylindrical paraxial wave equation, analogous to the Bessel beam solutions derived from the complete wave equation [30].

The LG functions have an effective radius defined as [31]

$$
\rho_{m}=w_{0} \sqrt{2 m+1} .
$$

The Fourier-Bessel transform of the LG function in Eq. (2) can be described with Eq. 7.421.1 of [32] as a similar LG function in frequency $k_{r}$ space. Just as $w_{0}$ defines a width parameter of the LG functions in Eq. (2), a corresponding value of $K=2 / w_{0}$ defines an equivalent parameter for the LG spectral description of Eq. (2). A radius, in $k_{r}$ space, can then be attributed to the LG spectrum in the same manner as Eq. (3), and making the intuitive assumption, based on [33], that this represents the maximum frequency at the center of the mode where the LG functions are obviously more oscillatory, a useful estimation of the transverse frequency at $r=0$ is predicted as

$$
\kappa_{m} \cong K \sqrt{2 m+1},
$$

which can be verified through comparison of $\psi_{m}\left(r_{0}\right)$ with the Bessel function $J_{0}\left(\kappa_{m} r_{0}\right)$ (see Fig. 2).

The Hermite-Gaussian and LG functions are orthogonal in the Cartesian and cylindrical coordinate systems, respectively, and an exact synthesis of a function $E_{0}\left(r_{0}\right)$ can be represented mathematically as an infinite sum of such functions weighted according to the expansion coefficients $A_{m}$. In this paper we will deal solely with cylindrically symmetric regimes, but the work has direct relevance to the application of the Hermite-Gaussian expansion in Cartesian systems. For practical applications of such expansions, a suitable choice of width parameter $w_{0}$ can allow the expansion to strongly converge to a function of finite radial extent using a truncated finite series of $M+1$ LG terms [23],

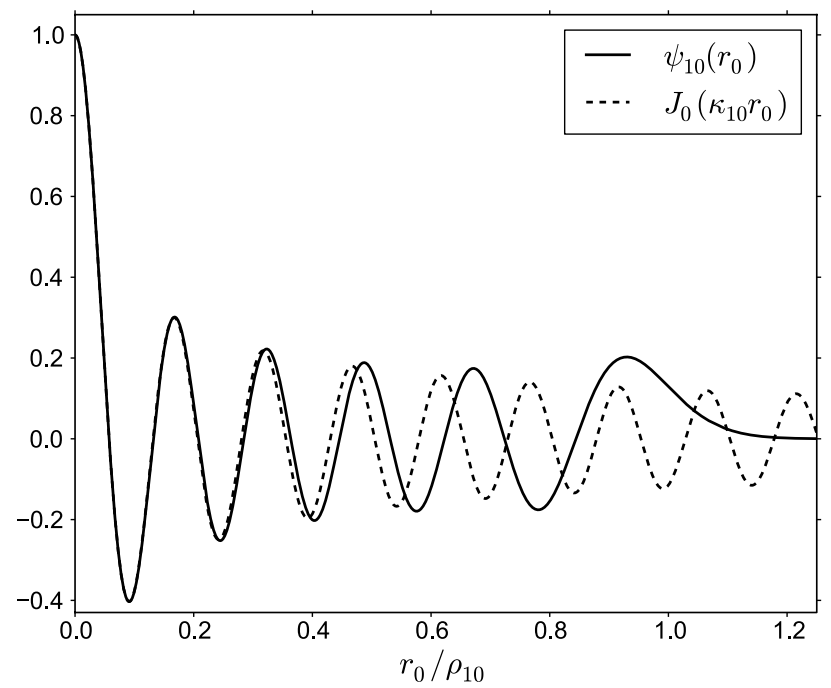

Fig. 2. Structure of the cylindrically symmetric LG function $\psi_{10}\left(r_{0}\right)$, from Eq. (2) without the normalization factor, compared with the Bessel function $J_{0}\left(\kappa_{10} r_{0}\right)$. The correlation between the Bessel and LG functions increases with larger values of $m$. 


$$
E_{0}\left(r_{0}\right) \cong \sum_{m=0}^{M} A_{m} \psi_{m}\left(r_{0}\right)
$$

where the expansion coefficient $A_{m}$ of a function $E_{0}$ is calculated by the integral

$$
A_{m}=\sqrt{\frac{2}{\pi w_{0}^{2}}} \int_{0}^{a} E_{0}\left(r_{0}\right) L_{m}\left(2 \frac{r_{0}^{2}}{w_{0}^{2}}\right) \exp \left(-\frac{r_{0}^{2}}{w_{0}^{2}}\right) 2 \pi r_{0} \mathrm{~d} r_{0}
$$

A finite-term expansion of a function with radius $a$ is possible with any real positive value of $w_{0}$, but with varying quality in the reconstruction. Borghi et al. showed that for a given value of $M$ the most accurate synthesis of a circular aperture function is obtained with [23]

$$
w_{0}=a / \sqrt{M}
$$

With an odd value of $M$, Eq. (7) imposes a width $w_{0}$ on the LG modes that creates exact equality between the radius of the aperture and the extent $\rho_{m}$ of the mode with the assumed median integer index $m=(M-1) / 2$ as given by Eq. (3). A similar relation exists for an even value of $M$ when the value of $\rho_{m}$ for the median integer index $m=M / 2$ corresponds, but not exactly, with $a$. Referring to the median index as $m=\mu$ for generality, application of Eq. (7) divides the finite mode set equally between modes that are not significantly truncated at $r_{0}=a$ with $m \leq \mu$, i.e., $\rho_{m} \leq a$, and those with $m>\mu\left(\rho_{m}>a\right)$, with the latter responsible for reconstruction of potential high frequency features around $r_{0}=a$ caused by a discontinuity at that point. Examples of the variation of $A_{m}$ in syntheses are shown in Fig. 3 with $M=49$ for a circular aperture of radius $a$, an annular aperture with a central circular obscuration of radius $a / 2$, and a hemispherical function $\left(a^{2}-r_{0}^{2}\right)^{1 / 2}$. The values of the coefficients generally alternate in sign according to $(-1)^{m}$, which is eliminated here for clarity of the trend $A_{m}$, and to generalize the value of the aperture radius $a$, the values are related to $w_{0}(2 \pi)^{1 / 2}$.

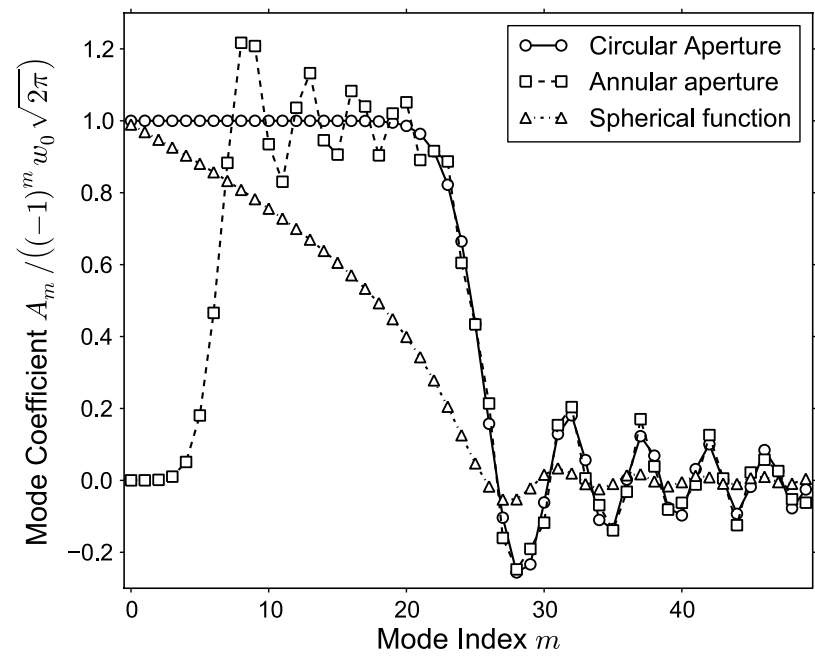

Fig. 3. Expansion coefficients for syntheses of a circular aperture, an annular aperture with inner radius $a / 2$, and a hemispherical function $\left(a^{2}-r_{0}^{2}\right)^{1 / 2}$. Calculated from Eq. (6) with $M=49$ and $w_{0}=a / M^{1 / 2}$.
A CW LG beam propagates in the paraxial regime according to $[\underline{24}, \underline{25}]$

$$
\begin{aligned}
\psi_{m}(\omega, r, z)= & \sqrt{\frac{2}{\pi w^{2}(\omega, z)}} L_{m}\left(\frac{2 r^{2}}{w^{2}(\omega, z)}\right) \\
& \times \exp \left(-\frac{r^{2}}{w^{2}(\omega, z)}\right) \exp \left(i \frac{\omega}{c}\left(z+\frac{r^{2}}{2 R(\omega, z)}\right)\right) \\
& \times \exp \left(-i(2 m+1) \tan ^{-1}\left(\frac{2 c z}{\omega w_{0}^{2}}\right)\right),
\end{aligned}
$$

with the sign of the phase terms adapted for consistency with related work in [22]. Diffractive spreading of the Gaussian modes is represented by an increase in the value of the beam width parameter $w$ with propagation distance $z$, described by

$$
w(\omega, z)=w_{0} \sqrt{1+\left(\frac{2 c z}{\omega w_{0}^{2}}\right)^{2}}
$$

with $w_{0}$ now representing the width parameter at the source plane (defined here to be at $z=0$ ). The width parameter $w(\omega, z)$ in Eq. (9) can also be applied, with Eqs. (3) and (ㅁ) giving $\rho_{m}(\omega, z)$ and $\kappa_{m}(\omega, z)$, identifying the mode's width and axial transverse frequency at any $z$ plane. The term $R(\omega, z)$ defines the radius of curvature of the diverging parabolic wavefront,

$$
R(\omega, z)=z\left(1+\left(\frac{\omega w_{0}^{2}}{2 c z}\right)^{2}\right)
$$

which has an infinite value (flat phase front) at $z=0$ and beyond the confocal range $z_{0}=\omega w_{0}^{2} /(2 c)$ converges to $R(z) \rightarrow z$. Using the above relations, a monochromatic field diffracted at an aperture can be approximated as a sum of LG beams weighted by the coefficients $A_{m}$, i.e.,

$$
E_{M}(\omega, r, z)=\sum_{m=0}^{M} A_{m} \psi_{m}(\omega, r, z),
$$

thus representing an asymptotic calculation of the Fresnel diffraction integral (FDI) [24,25].

Generally, however, illustration of the expansion for CWs is not presented with a clear explanation of the contributions made by each individual mode to the total calculated field, which can be difficult to identify in a CW model [23-29]. In the simulation of pulses as discussed here, the correlation between the LG basis functions and the expanded field is easier to illustrate, as distinct components of the diffracted fields are separated in time and space, allowing the contribution of each LG component to be identified, and subsequently it is shown how convergence to the FDI results is achieved through the propagation behavior of the individual modes. In our opinion, given the increased computational complexity in the practical simulation of pulse diffraction using the standard spectral method as given by Eq. (1), the application of such useful and efficient expansions is deserving of illustration in this field of study. 


\section{LAGUERRE-GAUSSIAN EXPANSION OF CIRCULARLY SYMMETRIC PULSED FIELDS}

The reduction of the FDI to an equivalent calculation as a finite sum of orthogonal modes with Eq. (11) can be used to greatly accelerate simulations of polychromatic fields. For paraxial calculation of pulsed fields, we can substitute the LG formulation given by Eq. (11) into Eq. (1), and an ultrashort field diffracted by an aperture can then be written as

$E_{M}(r, z, t)=\frac{1}{\sqrt{2 \pi}} \int_{-\infty}^{\infty} S(\omega)\left\{\sum_{m=0}^{M} A_{m}(\omega) \psi_{m}(\omega, r, z)\right\} \exp (-i \omega t) \mathrm{d} \omega$.

For application of Eq. (12) in the simulation of pulse modulation by dispersive lenses, for example, calculation of the mode coefficients is required for each individual frequency, which can be time consuming [34]. Nevertheless, once the coefficients have been determined, the field can then be efficiently determined as a finite sum, in contrast to the requirement of repeated integration over a highly sampled aperture function, as is required for the numerical solution of the FDI. In this report we assume the transmittance $E_{0}\left(r_{0}\right)$ to be amplitude modulating only, such as a basic circular or annular aperture, with the description of the aperture remaining constant at each frequency across the pulse's spectrum. The values of $A_{m}$ will then be equal for all frequency components, and we can then interchange the sum and integral in Eq. (12) to describe the pulsed field as

$$
E_{M}(r, z, t)=\sum_{m=0}^{M} A_{m} \Psi_{m}(\omega, r, z, t)
$$

where now the constituent basis terms $\Psi_{m}$ of the total field have the form of planar pulsed LG beams, described by

$$
\Psi_{m}(r, z, t)=\frac{1}{\sqrt{2 \pi}} \int_{-\infty}^{\infty} S(\omega) \psi_{m}(\omega, r, z) \exp (-i \omega t) \mathrm{d} \omega
$$

Thus, instead of an integration of diffracted monochromatic fields, as in Eqs. (1), (13) represents the diffracted field of a pulse by a superposition of individual and independently propagating LG "pulselets"- a term we use to distinguish the LG fields in Eq. (14) as basis components of the total diffracted pulse. A recent work applied a similar description to Eq. (13) with a small number of terms in a study of LG pulses with helical phase variations [35].

The type of LG pulse to be discussed in Section 3.A, i.e., Eq. (14), represents a short temporal excitation of an electric field with an amplitude defined by Eq. (2), generating a spectrum of individual monochromatic fields of different frequencies, each with the same transverse field profile at the plane $z=0$ (i.e., a constant value of $w_{0}$ for all frequency components). A distinction must be made between these planar pulsed fields [22] and the form of higher-order Gaussian pulses described in [36,37], which are the "isodiffracting" form of Gaussian pulses corresponding to the structures generated, for example, by the propagation of polychromatic fields within a mode-locked laser cavity. In the latter case, each chromatic Gaussian mode has a waist at $z=0$, but with different values of the waist parameter, i.e., $w_{0}(\omega)=$ $\left(2 c z_{0} / \omega\right)^{1 / 2}$, thus creating a constant confocal distance for each spectral component. Such fields are not directly comparable to the Gaussian pulses discussed here. Isodiffracting Hermite-Gaussian pulses have previously been discussed by Heyman and Beracha as basis modes in the expansion of "well-collimated" diffraction at an aperture [36]. However, the physical effects of the planar Gaussian pulses that we will discuss, e.g., the specific variations in group velocity and axial superluminal effects [22], do not relate to the distinct isodiffracting Gaussian modes, and the application of those fields as a basis set in [36] should not be confused as being an equivalent description to the procedure presented here.

We will illustrate the LG expansion technique for pulse propagation using the description given by Eq. (13), and in doing so we will describe the physical properties of individual planar Gaussian pulselets, exhibiting their role in the expansion of the total diffracted field. This, we suggest, gives a more intuitive description of the convergence of the LG expansion technique in the time domain. However, the reader should note that examples of the LG expansion to be given in Section 3.B are determined with significantly less computation time by the integration of CW fields, as in Eq. (12), representative of the general scheme outlined by Eq. (1). In the custom software used here, the LG functions are determined using the recurrence relation $L_{m+1}(r)=\left((2 m+1-r) L_{m}(r)-m L_{m-1}(r)\right) /$ $m+1$, with $L_{0}(r)=1$ and $L_{1}(r)=1-r$. Simulating the propagation of each individual LG pulselet with an index of $m+$ 1 can generate a significant amount of redundant data relating to LG functions of lower indices, 0 to $m$, that could alternatively be applied in a calculation involving Eq. (12), and with that saving considerable calculation time. For an increased efficiency in this method, analytical evaluation of Eq. (14) would allow for extremely swift evaluation of the expansion of pulsed fields, eliminating numerical integration in Eq. (14). Ziolkowski and Judkins, for example, established expressions for the propagation of ultrashort Gaussian beams [38]. Following the procedures in that report, it may be possible to derive similar expressions for the evaluation of the higher-order LG pulses in Eq. (14). Here, we will illustrate the propagation of the LG pulses through numerical calculations.

\section{A. Diffraction of Ultrashort Laguerre-Gaussian Beam Modes}

Toward a description of the propagation of an arbitrary ultrashort field as a superposition of LG pulselets, we first discuss the dynamics of the basis fields as independent optical structures. The behavior of both CW and pulsed Gaussian beams is prescribed by the well-known Gouy phase shift [33], which has been discussed in terms of its influence on the behavior of Gaussian and focused pulses [39-43]. Porras et al. have described the propagation of the fundamental $(m=0)$ planar Gaussian pulse and showed that at planes beyond the confocal distance of the central frequency, $z_{0}=\omega_{0} w_{0}^{2} /(2 c)$, such fields exhibit a group velocity greater than the vacuum speed of light $c$ [22]. Similar phenomena observed in the propagation of higher-order $(m>0)$ Gaussian pulses are directly related to those reported in [22], but the magnitudes of these propagation effects, such as a variation of group velocities and associated temporal delays, are increased in accordance with the larger value of the index $m$ within the Gouy phase term, associated with the wider transverse source fields. These phenomena will have importance in our modal description of a 
pulse diffracted by an aperture and the interpretation of each LG pulselet's role in such an expansion.

We describe the propagation of pulses with a conventional temporal Gaussian signal given as

$$
s(t)=\exp \left(-\ln 2 \frac{\left(t-t_{0}\right)^{2}}{\tau_{0}^{2}}\right) \exp \left(-i \omega_{0} t\right),
$$

where $\omega_{0}$ is the central angular frequency and $\kappa_{0}$ is the halfwidth at half-maximum (HWHM) of the Gaussian signal. The angular frequency spectrum of the signal in Eq. (15) is obtained by Fourier transformation and given by

$$
S(\omega)=\frac{\tau_{0}}{\sqrt{2 \ln 2}} \exp \left(-\tau_{0}^{2} \frac{\left(\omega-\omega_{0}\right)^{2}}{4 \ln 2}\right) .
$$

To generalize the signal for arbitrary frequencies, the fractional bandwidth of a pulse is given by $\gamma=\Delta \omega / \omega_{0}$, where $\Delta \omega=4 \ln 2 / \tau_{0}$ is the full width at half-maximum of the Gaussian frequency spectrum $S(\omega)$.

Figure $\underline{4}$ shows the propagation of a pulsed LG beam mode at various planes, as computed from Eq. (14). Note the structures of the fields near the axis in Figs. $\underline{4(\mathrm{c})}$ and $\underline{4(\mathrm{~d})}$, which bear obvious similarities to the pulsed form of Bessel beams, or "X-waves" [21]. In these plots, the axial
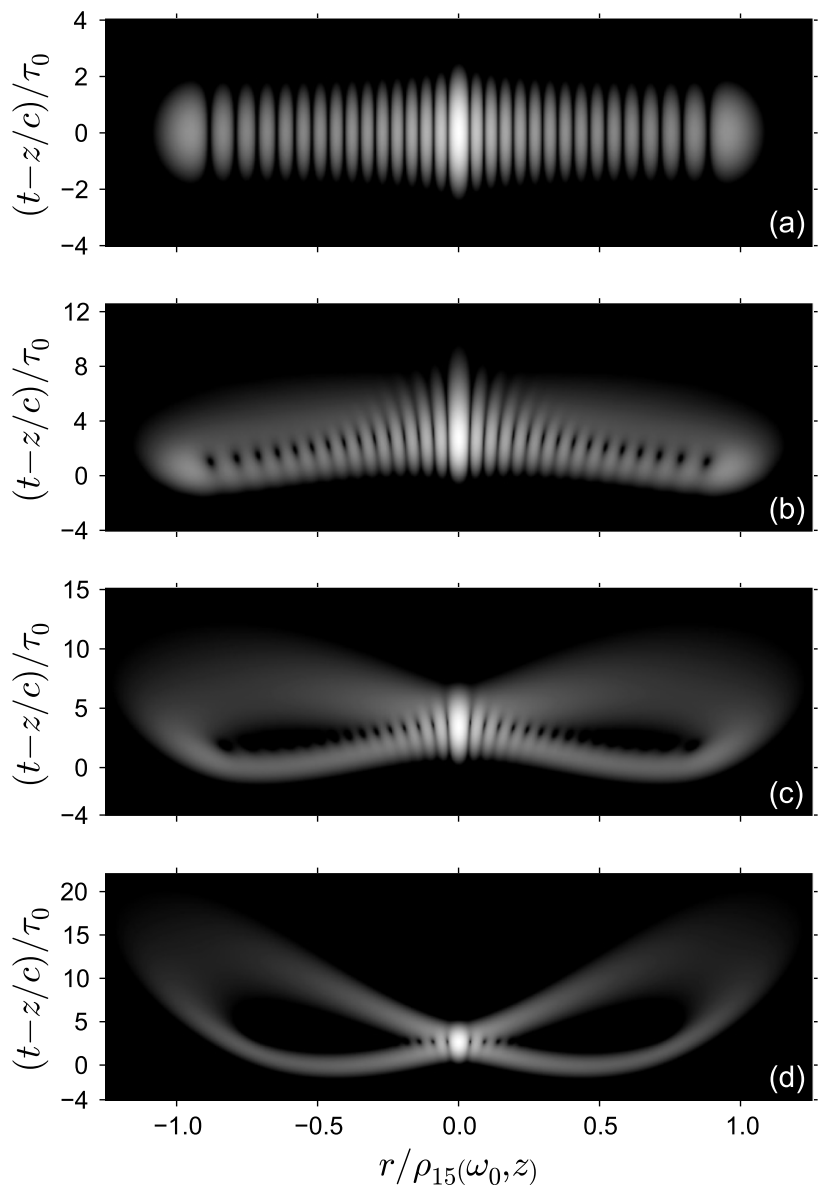

Fig. 4. Propagation of the $m=15$ planar Laguerre-Gaussian pulse $\left|\Psi_{15}(r, z, t)\right|^{2}$ from the numerical solution of Eq. (14). Calculated with $w_{0}=600 \lambda_{0}$ and a large bandwidth of $\gamma=0.6$ at the planes $z=$ (a) 0 , (b) $z_{0} / 2$, (c) $z_{0}$, and (d) $2 z_{0}$. The fields are displayed as a decibel scale representation of their normalized intensity between -35 and $0 \mathrm{~dB}$. extents are shown in relation to the mode's spot radius for the central frequency at each plane $\rho_{m}\left(\omega_{0}, z\right)$, while the temporal (vertical) axis is related to the detection time $(z / c)$ of an ideal plane wave pulse of equal duration, and in terms of the defined HWHM $\tau_{0}$ of the signal. For this paraxial description, the width of the field at $z=0$ is defined to be large in relation to the central wavelength $\lambda_{0}$. An analysis of the influence of diffraction on the spectral characteristics of the LG pulses is described in detail in [44].

While the plots of Fig. $\underline{4}$ show the general structure of the LG pulses with a significantly large bandwidth, such fields exhibit temporal dynamics that are not immediately apparent in Fig. 4 . These effects, relating to a changing pulse velocity and associated time delays, are more distinct in the axial propagation of pulselets with higher indices, as shown in Fig. $\underline{5}$, which displays the on-axis temporal amplitude structure of a pulsed LG beam at various planes. Modes with large values of $m$ such as those shown in Fig. 5, and much higher, can be required for an LG series description of diffraction at a hard aperture, particularly in the near field, where propagation can involve significantly large spatial frequencies.

From the suggestion of Porras et al. [22], and with relevance to the propagation features observed in Fig. $\underline{5}$, such as the varying velocities and the observed pulse broadening,
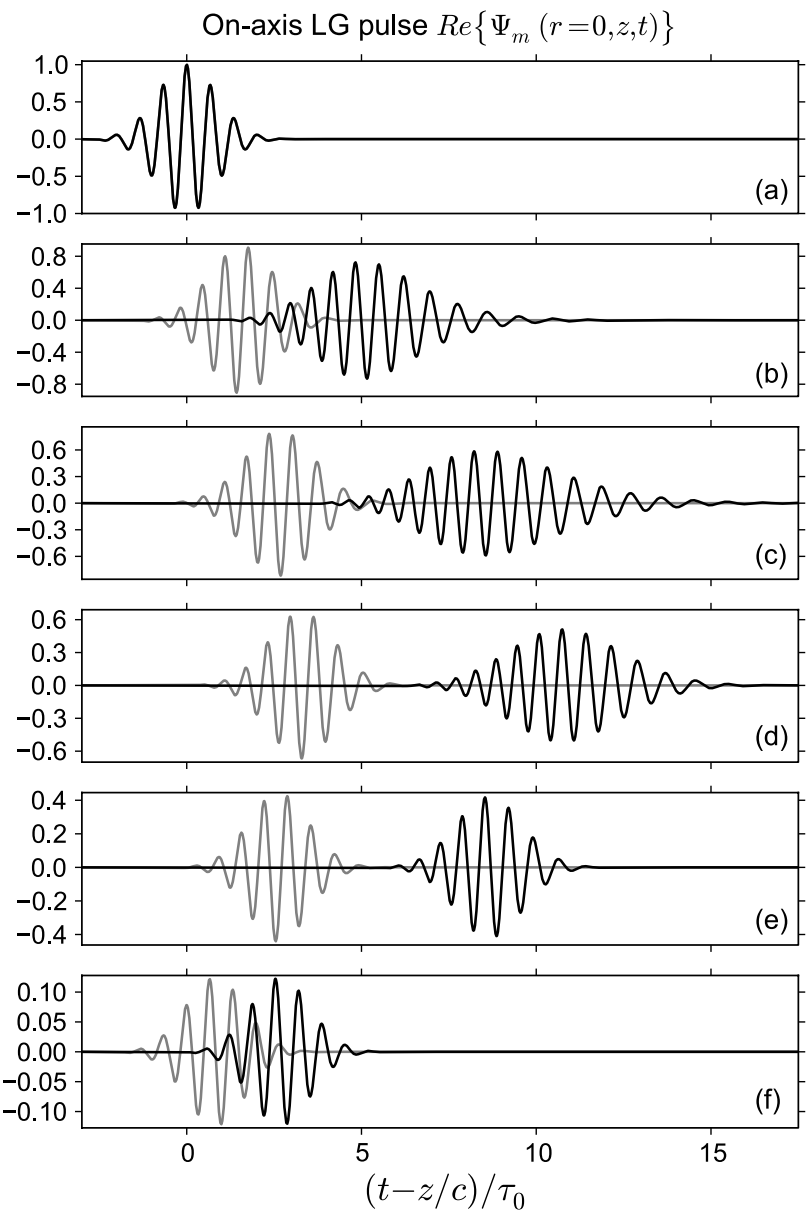

Fig. 5. Time-domain simulations for the on-axis temporal amplitude of the $m=30$ (gray) and $m=100$ pulsed LG modes $\operatorname{Re}\left\{\Psi_{m}(r=\right.$ $0, z, t)\}$ as simulated from Eq. (14). The fields are predicted at $z=$ (a) 0 , (b) $z_{0} / 4$, (c) $z_{0} / 2$, (d) $z_{0}$, (e) $2 z_{0}$, (f) $8 z_{0}$, with $w_{0}=300 \lambda_{0}$ and $\gamma=0.3$. 
an alternative description for the on-axis phase behavior of a pulsed LG beam is the analogy of a plane wave propagating through a hypothetical dispersive medium with a longitudinally varying refractive index $n_{G}(\omega, z)$, causing a phase variation equal to that created by the Gouy phase shift. A study of pulsed Bessel beams referred to a similar "spatially induced" dispersion [45], and this description is also parallel to the dispersion relation of waveguide modes that has been discussed in reference to pulse diffraction at an aperture [3] and in Young's double slit experiment [6]. For the axial $(r=0)$ variation of the fields, the phase $\phi$ of a monochromatic LG beam is given by

$$
\phi(\omega, z)=\frac{\omega}{c} z-(2 m+1) \tan ^{-1}\left(\frac{2 c z}{\omega w_{0}^{2}}\right),
$$

and we can write the local [46] on-axis longitudinal wavenumber as

$$
k_{z}(\omega, z)=\frac{\partial \phi(\omega, z)}{\partial z}=\frac{\omega}{c}-\frac{2 c(2 m+1)}{\omega w_{0}^{2}\left(1+\frac{4 c^{2} z^{2}}{\omega^{2} w_{0}^{4}}\right)} .
$$

For a monochromatic Gaussian beam the axial longitudinal phase velocity can be determined from $\omega / k_{z}$. For polychromatic pulses an effective Gouy "refractive" index $n_{G}(\omega, z)$ can be represented as a multiplicative factor on the wavenumber $\omega / c$, i.e., $n_{G}(\omega, z) \omega / c=k_{z}(\omega, z)$, and a dispersion relation for the axial Gouy phase shift may then be written as

$$
n_{G}(\omega, z)=1-\frac{2 c^{2}(2 m+1)}{\omega^{2} w_{0}^{2}\left(1+4 \frac{c^{2} z^{2}}{\omega^{2} w_{0}^{4}}\right)} .
$$

Combining Eq. (19) with the common formula for group velocity in dispersive media [47],

$$
v(z)=\frac{c}{n_{G}\left(\omega_{0}, z\right)+\left.\omega_{0} \frac{\partial n_{G}(\omega, z)}{\partial \omega}\right|_{\omega=\omega_{0}}},
$$

the on-axis group velocity can then be expressed for an LG pulse with $\gamma \ll 1$ as

$$
v_{m}(z)=c\left(1-\left(m+\frac{1}{2}\right) w_{0}^{2} \frac{\left(z^{2}-z_{0}^{2}\right)}{\left(z^{2}+z_{0}^{2}\right)^{2}}\right)^{-1},
$$

which with $m=0$ is equivalent to Eq. (6) in [22]. Figure 6 illustrates the axial group velocity of the LG pulse modes from Eq. (21).

On the optical axis, the time delay of an LG pulsed mode in relation to a plane wave pulse, $T_{m}(z)$, can be determined at a plane $z$ by accounting for the variation of the group velocity of the LG field at each previous plane, $z^{\prime}<z$, i.e.,

$$
T_{m}(z)=\left[\int_{0}^{z} \frac{\mathrm{d} z^{\prime}}{v_{m}\left(z^{\prime}\right)}\right]-\frac{z}{c}=(2 m+1) \frac{2 c z w_{0}^{2}}{4 c^{2} z^{2}+w_{0}^{4} \omega_{0}^{2}}
$$

Yang et al. have derived an equivalent expression to Eq. (22) for off-axis points and describe the variation in the axial carrier envelope phase of the LG pulses [42], while the associated spatial shift is described in [43]. The variation

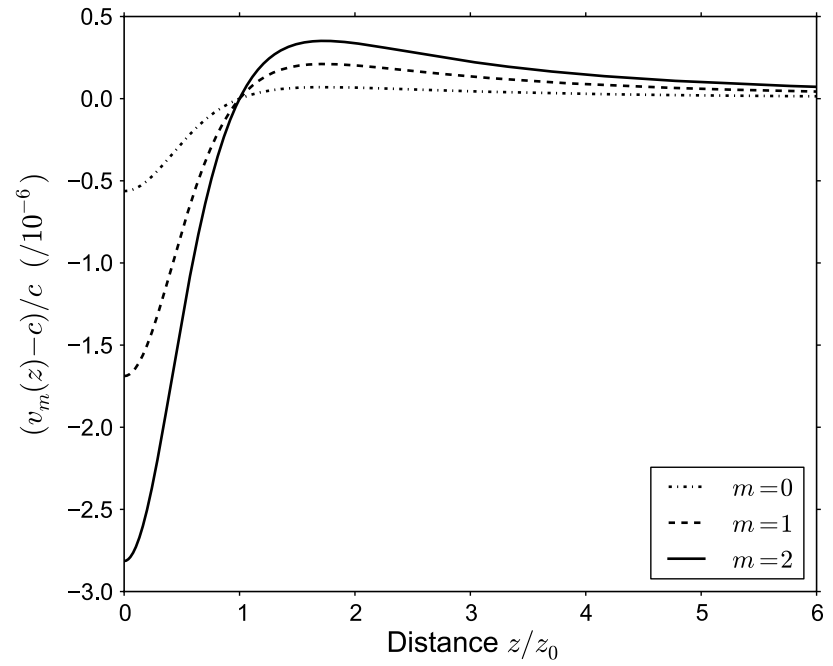

Fig. 6. Group velocity, $v_{m}(z)$, of the planar Laguerre-Gaussian pulses of different indices, with $\gamma=0.1$ and $w_{0}=300 \lambda_{0}$.

of $T_{m}(z)$ is shown in Fig. 7 , with a maximum delay occurring at the confocal range $z_{0}$ with a magnitude of

$$
T_{m}\left(z_{0}\right)=(m+1 / 2) / \omega_{0}
$$

Analytical descriptions for the effects of dispersion are conventionally derived through a Taylor series representation of the wavenumber $\omega n(\omega) / c$. For example, the expression of group velocity given by Eq. (20) is related to the inverse of the first two terms of such a series expansion, while the secondorder terms can be applied to account for pulse broadening attained in a dispersive medium [47-49]. Here, we identify from numerical calculations the second-order effects of a free-space Gouy dispersion, which are clearly observable in Fig. 5 with an increased axial duration of the pulses, most obvious in the case of $m=100$. This free-space broadening is physically related to a similar effect observed in the diffraction of a pulse at a finite circular aperture [19], which in that case is shown to occur due to the axial interference of the BW

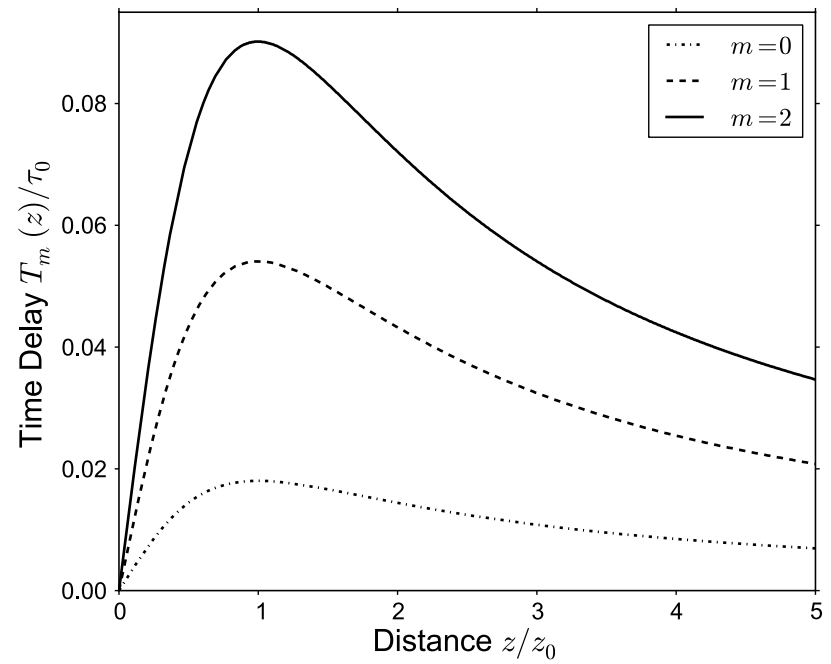

Fig. 7. Axial time delay, $T_{m}(z)$, of the temporal amplitude envelope of the pulsed Laguerre-Gaussian modes $\left|\Psi_{m}(r=0, z, t)\right|$ in relation to the half-duration of the pulse $\tau_{0}$, with $w_{0}=300 \lambda_{0}$ and $\gamma=0.1$. 
and GW features. The increased axial duration of a pulse at a plane $z>0$ can be accounted for by the longer time required for a field source at $r_{0}>0$ to arrive at an axial point than a source at $r_{0}=0$. In the case of diffraction at a hard aperture, the axial broadening of a pulse involves a splitting of the axial field into boundary and geometric features due to the abrupt discontinuity at the aperture edge (c.f. Figs. 2-4 of [19]). This is in contrast to the related effects shown in Fig. 5 , where the axial pulse structure remains within a single envelope, attributable to the continuity of the LG source along $r_{0}$. From numerical evaluation of Eq. (14), Fig. $\underline{8}$ quantifies the free-space broadening in the variation of the temporal HWHM, $\tau(z)$, of the axial LG pulse envelope $\left|\Psi_{m}(r=0, z, t)\right|$ in relation to the HWHM $\tau_{0}$ of the Gaussian time signal at $z=0$. Although such a temporal broadening is faintly visible in the $m=0$ planar Gaussian pulse, as shown in Fig. 4 of [22], the authors of this paper are not aware of any previous analysis of this axial broadening in planar Laguerre-Gaussian pulses. As is evident in the magnitude of the broadening as presented in Fig. 8, the effect is not an obvious feature in such fields of lower indices as studied in $[\underline{22}, \underline{42}, \underline{43}, \underline{48}]$, which did not address this subtle characteristic of planar LG pulse propagation.

It can be seen that the axial duration of the planar Gaussian pulses is not a constant in free space but broadens to a maximum value and at greater distances decreases toward a value slightly greater than $\tau_{0}$, which we assume to be related to the convergence of the Gouy phase shift in $z>z_{0}$. From numerical data as in Fig. $\underline{8}$, with increasing values of $m$ the plane at which a maximum axial duration $\tau_{m}(z)$ occurs is found to converge to

$$
z=z_{0} / \sqrt{3}
$$

This is compared to the plane of the maximum value of the group velocity $v_{m}$ at $z=\sqrt{ } 3 z_{0}$ [22], which is independent of the index $m$. Although we do not give an analytical description of these broadening effects here, the plane identified by Eq. (24) can be shown to correspond to that at which an analogous Gouy group velocity dispersion (GVD; see, e.g., [47-49]) alternates from a negative to a positive value. The

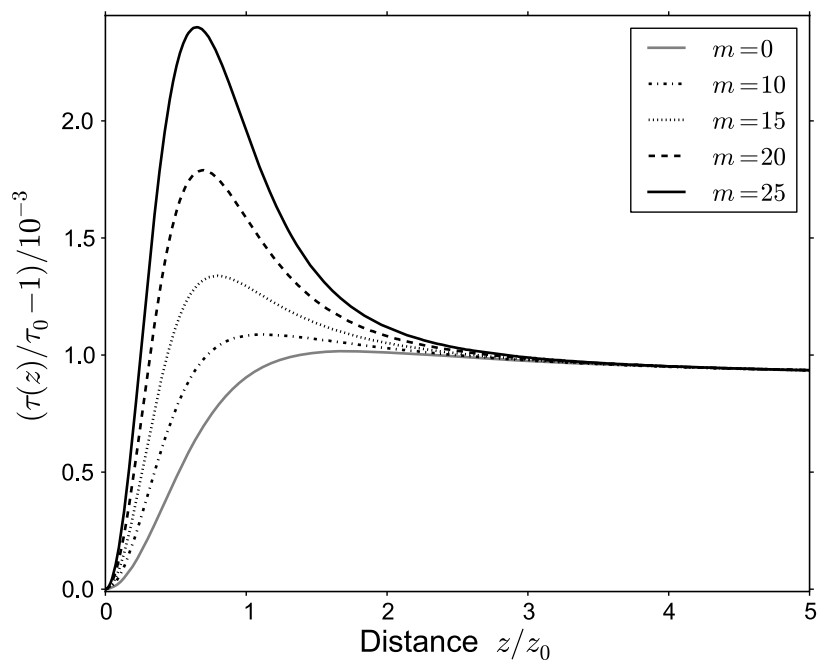

Fig. 8. Variation in the HWHM $\tau(z)$ of the on-axis temporal field $\left|\Psi_{m}(r=0, z, t)\right|$ at each plane $z$ related to the half-duration $\tau_{0}$ of the source signal defined at $z=0 . w_{0}=300 \lambda_{0}$ and $\gamma=0.1$. phenomena shown in Fig. 8 may perhaps be quantified analytically in future work using the description of the Gouy shift in Eq. (19) alongside conventional expressions relating to second-order effects of dispersion. It may also be worth pointing out that in Fig. $\underline{5}$ the on-axis LG pulses show the formation of a negative chirp in free space within $z<z_{0} / \sqrt{ } 3$ due to a negative value of a Gouy GVD, which is in contrast to the effects conventionally observed with normal material dispersion $[48,49]$. The chirp structure in Fig. $\underline{5}$ is also a feature of pulse diffraction at an aperture with a finite width and depth that introduces spatial dispersion [3]. We must emphasize that in Fig. , although $\tau(z)$ is illustrated in relation to the HWHM of the Gaussian envelope, $\tau_{0}$, the temporal field on the axis does not retain an ideal Gaussian envelope in the near field with an obvious asymmetry introduced in a similar man-

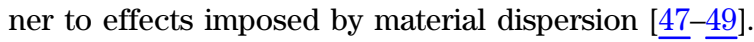

The free-space Gouy dispersion also influences the axial amplitude of the Gaussian pulses. Normalized to its value at $z=0$, the on-axis amplitude of a CW LG beam exhibits a variation described by $w_{0} / w(\omega, z)$, independently of the mode index. For pulsed LG modes, there is a variation in this description related to the axial broadening of the pulselets as shown in Fig. . The deviation of the amplitude falloff is depicted in Fig. 9 , which describes the maximum amplitude of the pulse envelope at each $z$ plane. This is again a small variation with lower indexed pulselets; thus, to emphasize the effect, the propagation of planar LG pulse modes with large indices and a significant bandwidth was computed.

Figures $\underline{8}$ and $\underline{9}$ highlight the restriction of these diffraction effects to the confocal region, with the pulselets attaining a more "standard" behavior at planes beyond $z_{0}$.

\section{B. Numerical Results for the LG Expansion of Diffracted Optical Pulses}

Following the LG expansion for continuous fields as outlined by Eq. (11), we can simulate paraxial diffraction of a pulse by superimposing a series of LG pulselets in a similar manner, as in Eq. (13), and illustrate the relation of the physical behavior of the $\overline{L G}$ pulselets to the convergence of the expansion. The

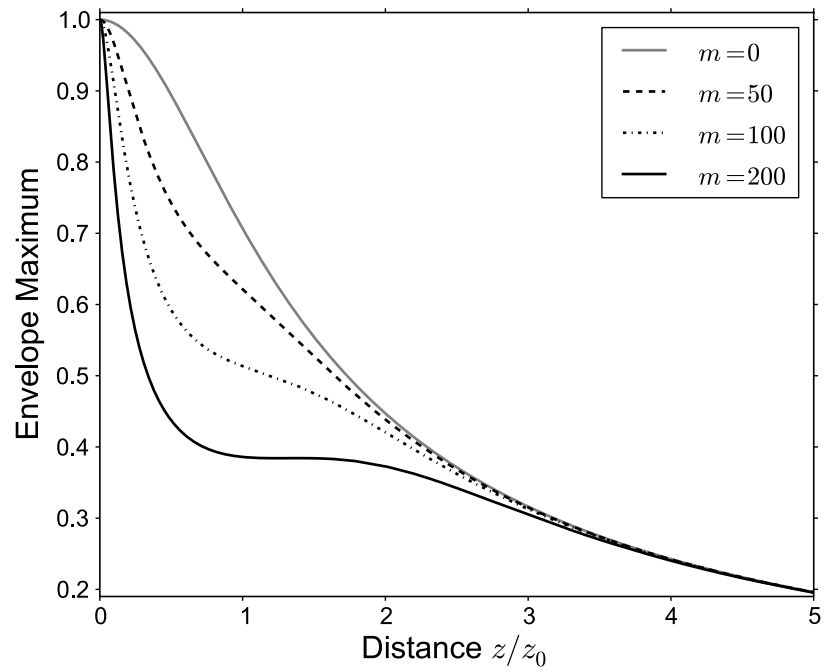

Fig. 9. Maximum value of $\left|\Psi_{m}(r=0, z, t)\right|$ at each plane $z$, with $\gamma=0.3$ and $w_{0}=300 \lambda_{0}$. The on-axis amplitude of the $m=0$ pulsed LG mode varies negligibly on this axis scale from $w_{0} / w\left(\omega_{0}, z\right)$. 
effects of the axial group velocity of each mode $v_{m}(z)$ and the associated time delays $T_{m}(z)$ are important issues in this context. To illustrate the expansion, we describe the method in simulation of the diffraction of a plane wave pulse at a circular aperture of radius $a$. Despite its simplistic form, such an aperture can be a difficult structure to represent mathematically with a finite convergent series technique due to the discontinuity at the aperture boundary [23,28] (recall Gibb's phenomenon in Fourier theory). Figures 10(a)-10(c) show the predicted field amplitudes $\operatorname{Re}\left\{E_{M}(r=\overline{0, z, t})\right\}$ from an LG expansion at the optical axis for mode sets of various sizes, while Figs. $10(\mathrm{~d})-10(\mathrm{f})$ show the same expansions in two dimensions as $\left|E_{M}(r, z, t)\right|^{2}$. It should be recalled throughout this section that the width parameter $w_{0}$ is adapted according to Eq. (7) for each $M+1$ term expansion.

On the optical axis, acceptable reconstruction of the desired field is obtained only with mode sets that contain pulse modes for which the associated Gouy dispersion effect creates a temporal delay that approaches or exceeds that of the predicted axial BW, i.e., greater values of $M$. In Fig. 10(b) the truncation of the series expansion to a finite number of terms can be seen to cause the pulse mode with $m=M$ to become significantly more obvious, in that case with $T_{M} \approx 5 \tau_{0}$ and in Fig. 10 (c) with $T_{M} \approx 10 \tau_{0}$. Only with higher values of $M$ do the location and magnitude of such a deviation from the FDI result become less intrusive in the region of interest due to a greater delay $T_{M}$ and a smaller value of the coefficient $A_{M}$.

Illustrations of the convergence of the LG expansion of a circular aperture function can be found in [23], and a similar description relating to the Hermite-Gaussian expansion is given in [28]. However, those examples apply only to the syntheses of the aperture functions and do not exhibit the convergence of the expansion in relation to the diffracted field beyond the aperture. To offer a simple exhibition of axial convergence in the LG expansion of pulses, we can compare the results with the equivalent FDI calculation for a circular aperture, $E_{F}$, through calculation of the normalized mean square error (NMSE):

$$
\mathrm{NMSE}=100 \times \frac{\int_{t}\left|\operatorname{Re}\left\{E_{F}(0, z, t)\right\}-\operatorname{Re}\left\{E_{M}(0, z, t)\right\}\right|^{2} \mathrm{~d} t}{\int_{t}\left|\operatorname{Re}\left\{E_{F}(0, z, t)\right\}\right|^{2} \mathrm{~d} t} .
$$

Figure 11 shows the variation of the NMSE at four planes $z=a^{2} /\left(\bar{N}_{0} \lambda_{0}\right)$, where $N_{0}$ is the Fresnel number.

In Fig. 11 the errors calculated with $N_{0}=15,10$, and 5 show a suitable convergence to the FDI result with smaller values of $M$ than in the case of $N_{0}=20$. This is obviously due to the smaller time separation between the GW and BWs on the axis at distances farther from the aperture where the lesser delay $T_{m}$ of LG modes with smaller indices can be sufficient to accurately represent the features of the on-axis BW. The calculated errors of around 50\% shown in the data represent suitable syntheses of the on-axis GW only, which has approximately equal energy to that of the axial BW feature at the planes described.

Now, to make a correlation between the propagation of the individual LG pulselets and the field that they expand, we can use Eq. (4) to imply the contributions of specific LG pulselets to the structure of the total diffracted field. This is not a consideration in previous studies of the orthogonal expansions for CW simulation [23-29], and here, with pulsed fields, the involvement of the individual LG modes in the field expansion can be visualized more clearly, specifically in the formation of the BWs.

It was explained in $[14,15]$ that in a cylindrically symmetric system, close to the optical axis, the BW can be represented by zeroth order Bessel pulses, related to X-waves [16,21]. The spherical and parabolic/paraxial waves generated at the boundary of an aperture at $r_{0}=a$ are detected on the optical axis at a plane $z$, in relation to a plane wave pulse, at the respective retarded times $[\underline{14}, \underline{19}]$
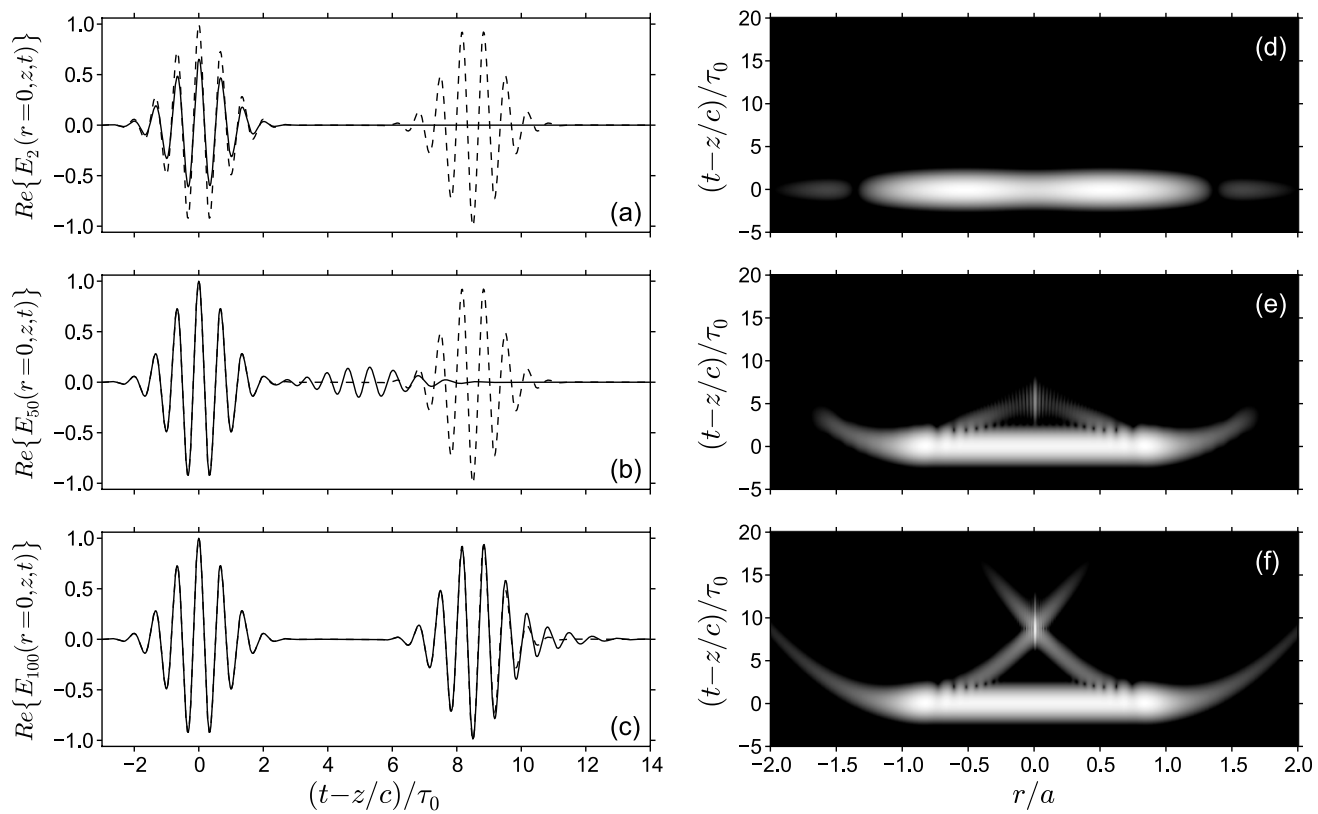

Fig. 10. (a)-(c) Axial convergence of an LG pulse expansion (solid curve) toward the result obtained with the conventional FDI (dashed curve) for a pulse diffracted at a circular aperture with $M=$ (a) 2, (b) 50, (c) 100. (d)-(f) A two-dimensional (1 + 1D) intensity representation $\left|E_{M}(r, z, t)\right|^{2}$ of the respective data in (a)-(c) within -40 and $0 \mathrm{~dB}$. The fields are predicted with a fractional bandwidth of $\gamma=0.3$ at $z=a^{2} /\left(25 \lambda_{0}\right)$. 


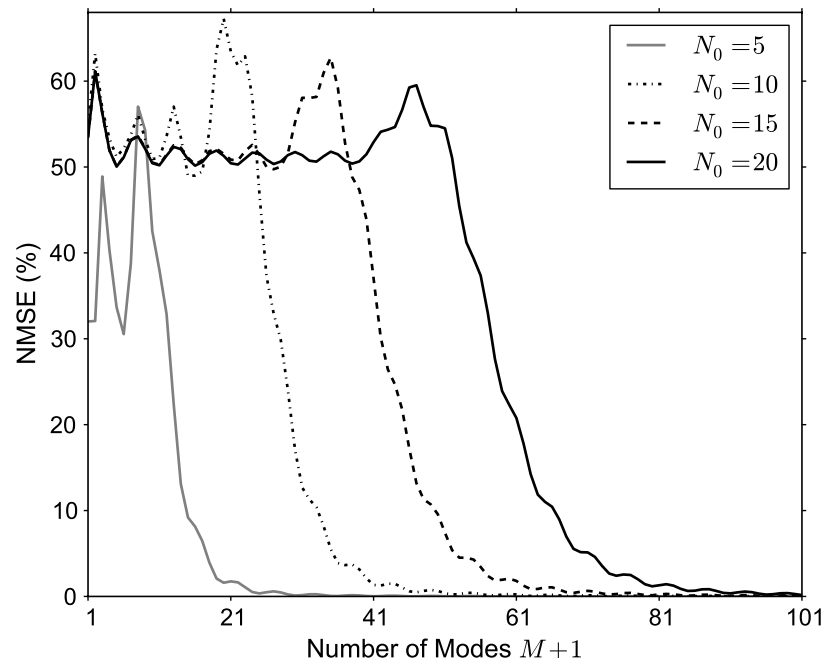

Fig. 11. Normalized mean square error (NMSE) between the LG expansion $\operatorname{Re}\left\{E_{M}(r=0, z, t)\right\}$ and the corresponding FDI calculation of the on-axis field of a $\gamma=0.3$ plane wave pulse diffracted by a circular aperture with various values of the Fresnel number $N_{0}$. For the case of $M=0$, we assumed a value of $w_{0}=a$.

$$
T^{s}(z)=\frac{\sqrt{a^{2}+z^{2}}}{c}-\frac{z}{c} \quad \text { and } \quad T^{p}(z)=\frac{a^{2}}{2 c z},
$$

and from $(d(T(z)+z / c) / d z)^{-1}$ the associated on-axis velocities are

$$
v^{s}(z)=\frac{c \sqrt{a^{2}+z^{2}}}{z} \quad \text { and } \quad v^{p}(z)=-\frac{2 c z^{2}}{a^{2}-2 z^{2}},
$$

where the superscripts $s$ and $p$ denote the respective spherical and parabolic/paraxial representations. The BW has a transverse frequency at $r=0$ of

$$
k_{r}^{s}(z)=\frac{k_{0} a}{\sqrt{a^{2}+z^{2}}} \quad \text { and } \quad k_{r}^{p}(z)=\frac{k_{0} a}{z},
$$

where $k_{0}=\omega_{0} / c$ is the central wavenumber. The normalized transverse intensity profile of the BW close to the axis then approaches the function $\left|J_{0}\left(k_{r} r\right)\right|^{2}$, with greater correlation found for longer pulses with $\gamma \rightarrow 0$ [14]. In an LG expansion with a large value of $M$, in the propagation of the $\mu$ th mode at greater distances from the aperture plane, depending on $M$ and subsequently $w_{0}$, the group velocity $v_{\mu}$, Eq. (21), time delay $T_{\mu}$, Eq. (22), and on-axis transverse frequency $\kappa_{\mu}\left(\omega_{0}, z\right)$, from Eq. (4) with $K\left(\omega_{0}, z\right)=2 / w\left(\omega_{0}, z\right)$, converge upon the corresponding values of those parameters for the BWs as given by Eqs. (26). For example, the far-field divergence angle of a Gaussian beam in relation to an optical axis is given by $\theta=2 /\left(k_{0} w_{0}\right)$, and an asymptotic value of $w\left(\omega_{0}, z \gg z_{0}\right)$ is then $w\left(\omega_{0}, z\right)=z \theta$, with the conventional paraxial approximation of $\theta \approx \tan \theta$. Assuming an odd value of $M$, the value of $\kappa_{\mu}\left(\omega_{0}, z\right)$ from Eq. (4) with $\mu=(M-1) / 2$ is then given as

$$
\kappa_{\mu}\left(\omega_{0}, z\right)=\frac{k_{0} w_{0}}{z} \sqrt{2\left(\frac{M-1}{2}\right)+1}=\frac{k_{0} a}{z}, \quad \text { for } z \gg z_{0}
$$

after substitution of $w_{0}$ from Eq. (7), equating the axial transverse frequency of the $m=\mu$ mode to that of the paraxial BW in (26c). Thus, applying the LG expansion in paraxial regions, the $m=\mu$ pulselet dominates the representation of the physical Bessel BW structure (although it is the interference of the pulselets with indices surrounding $m=\mu$ that creates the prominence of the BW feature). Equation (27) is specifically a calculation for monochromatic beams, but the valid assumption is made here that the transverse frequency of the LG pulselets at $r=0$ will vary negligibly with $\gamma \ll 1$ from that of the $\mathrm{CW}$ mode with angular frequency $\omega_{0}$. The correspondence between the LG pulse modes and the BWs is also more evident in expansions with larger values of $M$, which allows for accurate reconstruction closer to the aperture plane, where BWs are more obvious with greater separation from the GW.

To graphically highlight this relationship between the BWs and the LG pulselets in an expansion, we show the diffraction of a plane wave pulse at a simple binary grating described by

$$
E_{0}\left(r_{0}\right)=\left\{1+\operatorname{sgn}\left[\cos \left(2.5 \pi r_{0} / a\right)\right]\right\} / 2,
$$

which has two transparent regions within $r_{0}=\{0,0.2 a\}$ and $\{0.6 a, a\}$, and an opaque intermediate region. Figure 12 shows the distribution of $A_{m} /(-1)^{m}$ for the LG expansion of this function with $M=499$. With three dislocations across the aperture function in Eq. (28), the distribution of the coefficients $A_{m}$ is correspondingly truncated at three values of $m$, as indicated in Fig. 12. The indices with $m=9,90$, and 249 are identified, with the radii $\rho_{m}$ of the LG functions $\psi_{m}\left(r_{0}\right)$ corresponding to the discontinuities of the aperture (exactly in the case of $m=249$ with $\rho_{249}=a$ ). For these specified values of $m$, the associated LG pulselets are then seen in an expansion to become more pronounced than those with indices of a similar value but smaller values of $A_{m}$, and they subsequently form the Bessel BWs related to those points.

In Fig. 13 the pseudocolor plot shows the predicted structure of a plane wave pulse diffracted at the aperture defined in Eq. (28), and the overlaid contour lines show the locations and general structures of the component $m=0,9,90$, and 249 (= $\mu$ ) LG pulse modes. For the regions shown in Fig. 13(b), NMSE errors of $19.39 \%, 3.41 \%$, and $0.33 \%$ were found between the LG expansions with respective values of $M=20,50$, and 100 in comparison with results of the FDI. For Fig. 13(a)

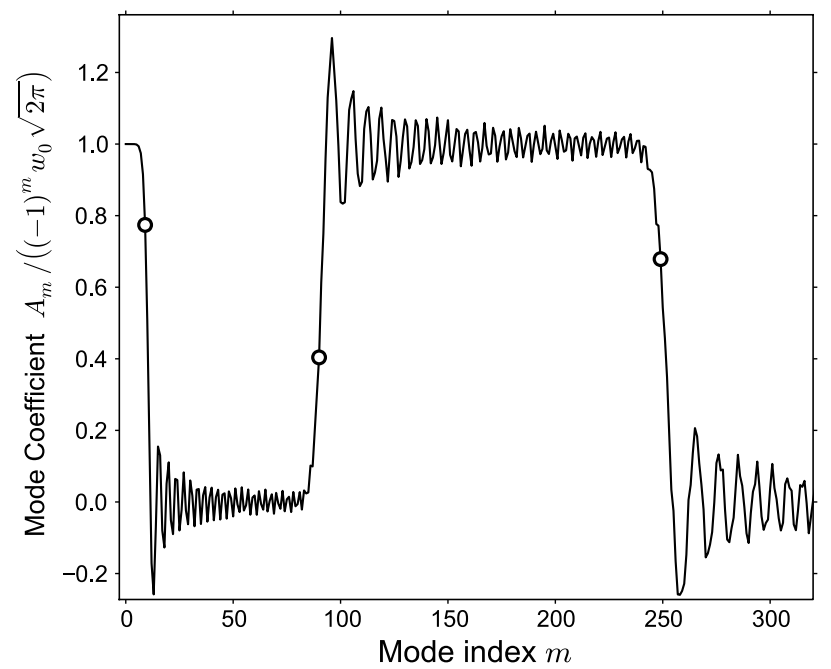

Fig. 12. LG expansion coefficients for the grating structure defined by Eq. (28), with $M=499$. Recall that the mode indices have discrete integer values only. 

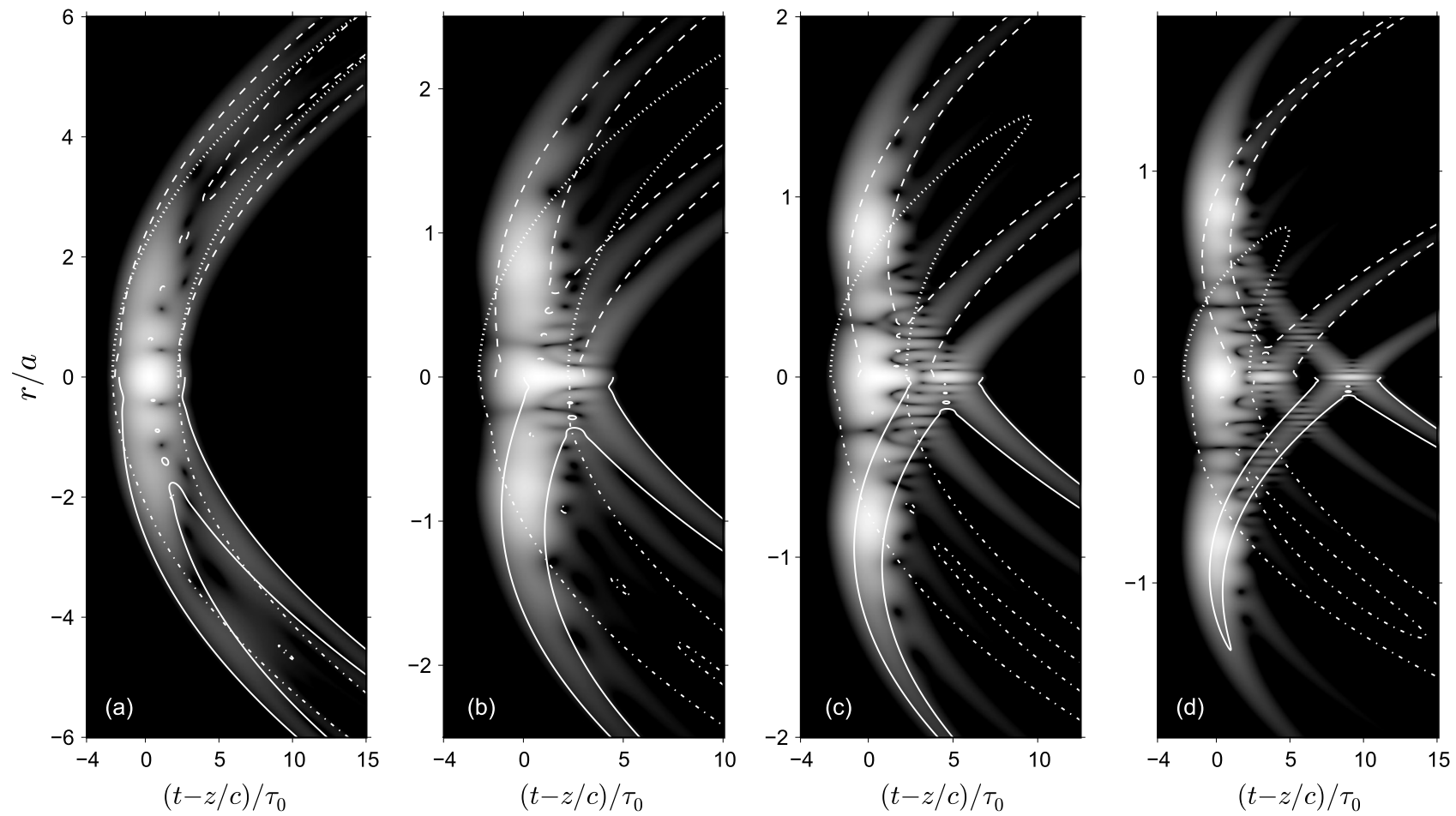

Fig. 13. Paraxial LG expansion calculation, with $M=499$, of a plane wave pulse with a $\gamma=0.4$ Gaussian spectrum diffracted by the annular aperture structure defined by Eq. (28). The fields are calculated with the Fresnel number $N_{0}=$ (a) 1 , (b) 5 , (c) 10 , (d) 20 . The $-30 \mathrm{~dB}$ contours of the $m=0$ (dotted curve), $m=\overline{9}$ (dash-dotted curve), $m=90$ (dashed curve), and $m=249$ (solid curve) component LG pulselets are superimposed and are shown on alternating sides of $r=0$ for clarity of the total field. The intensity data is shown on a normalized logarithmic $\mathrm{dB}$ scale between -40 and $0 \mathrm{~dB}$, except in (a), which has a lower limit of $-50 \mathrm{~dB}$.

the corresponding NMSE errors are $0.08 \%, 0.003 \%$, and $0.00014 \%$, which proved to be very efficient when using Eq. (12), which was actually applied in Fig. 13 for greater efficiency in generating such high resolution plots of $751 \times 1001$ points.

The intuitive description given by Fig. 13 of the formation of a diffracted field with a clear illustration of the role of the individual modes cannot be so easily interpreted from the application of an LG expansion in CW simulations.

\section{CONCLUSION}

We have illustrated the application and convergence of the LG expansion in relation to optical pulses. In practice, the scheme by which we have discussed the LG expansion, i.e., Eq. (13), is not the most computationally efficient procedure, but it was used to intuitively explain the convergence of the series expansion in the time domain through a description of individual LG pulses. In relation to this, application of analytical expressions for the propagation of such planar pulses, as derived for the fundamental Gaussian beam in [38], may allow for an extremely efficient computational procedure, removing the requirement of numerical integration in Eq. (14).

The diffraction of higher-order Gaussian pulses has been simulated numerically and shows properties such as superluminal group velocities and related delays, which are increased from the same effects of the $m=0$ Gaussian pulse studied previously [22]. The second-order effects of a spatial Gouy dispersion, i.e., an axial pulse broadening, have been identified through simulations. A variation in the axial amplitude of pulsed Gaussian beams imposed by the dispersion effect has been described and shows a deviation from the axial amplitude of the CW LG beams. These features have greater significance for LG pulses with larger indices and bandwidths.

Based on their physical characteristics, the structure of higher-order LG pulses has been shown in an expansion to represent the paraxial form of the BWs typically observed in pulse diffraction at an aperture, allowing for an alternative interpretation of the physical effects shown in [14].

Calculation of paraxial diffraction effects using series techniques such as the LG method discussed here, the WenBreazeale method [50,51], and similar expansions presented recently by Zamboni-Rached et al. [52,53] has an advantage over direct solution of the FDI (using a numerical sum or trapezoidal rule method) when the number of required series expansion terms (modes) is less than the number of aperture samples needed for convergence of the FDI, which can be very large for complicated apertures. As was said of similar finite series expansions in [52], "One can get in a few seconds, or minutes, high-precision results that could otherwise require lengthy numerical simulation." An important practical aspect of the series expansion techniques, in contrast to direct solution of the FDI, is that they can take advantage of efficient matrix and vector techniques available in numerical software such as Python's Numpy module, MATLAB, Octave, and R, for example. Using vectorization, the field at multiple points at a constant plane can be calculated without an extremely significant increase in computation time from a similar operation at an individual point (obviously depending on the array dimensions).

With such series expansions, the diffractive influence of an aperture is represented in the variation of the expansion 
coefficients. For a simple increase in efficiency for the simulation of CWs, the two-dimensional $(r, z)$ form of the propagating modes can be predefined in digital memory without reference to any aperture function and used with different variations of $A_{m}$ for repeated analyses of small variations in aperture structures, for example. Any difference in the aperture radius can be accounted for by adapting $w_{0}$ according to Eq. (7) and appropriately scaling the transverse and longitudinal ranges of the predefined modes with respect to $w_{0}$ and $z_{0}$.

The FDI is one of the most widely applied techniques in Optics beyond the geometrical regime. However, its direct solution remains computationally intensive, which is greatly augmented in the simulation of optical pulses, particularly in generating two-dimensional representations of fields. Despite the fact that they are common knowledge in optics, we believe that finite series techniques such as the HermiteGaussian and LG expansions have not reached their full potential in the field. The efficiency offered by these methods is suggested to be extremely beneficial to any researcher frequently requiring diffractive analyses of arbitrary apertures and lenses.

\section{REFERENCES}

1. M. Schultze, A. Wirth, I. Grguras, M. Uiberacker, T. Uphues, A. J. Verhoef, J. Gagnon, M. Hofstetter, U. Kleineberg, E. Goulielmakis, and F. Krausz, "State-of-the-art attosecond metrology," J. Electron Spectrosc. Relat. Phenom. 184, 68-77 (2011).

2. M. F. Kling and M. J. J. Vrakking, "Attosecond electron dynamics," Annu. Rev. Phys. Chem. 59, 463-492 (2008).

3. R. M. Joseph, S. C. Hagness, and A. Taflove, "Direct time integration of Maxwell's equations in linear dispersive media with absorption for scattering and propagation of femtosecond electromagnetic pulses," Opt. Lett. 16, 1412-1414 (1991).

4. S. Nekkanti, D. Sullivan, and D. S. Citrin, "Simulation of spatiotemporal terahertz pulse shaping in 3-D using conductive apertures of finite thickness," IEEE J. Quantum Electron. 37, 1226-1231 (2001)

5. P. M. Goorjian and A. Taflove, "Direct time integration of Maxwell's equations in nonlinear dispersive media for propagation and scattering of femtosecond electromagnetic solitons," Opt. Lett. 17, 180-182 (1992).

6. A. M. Nugrowati, S. F. Pereira, and A. S. van de Nes, "Near and intermediate fields of an ultrashort pulse transmitted through Young's double-slit experiment," Phys. Rev. A 77, 053810 (2008).

7. A. Gürtler, C. Winnewisser, H. Helm, and P. U. Jepsen, "Terahertz pulse propagation in the near field and the far field," J. Opt. Soc. Am. A 17, 74-83 (2000).

8. E. Budiarto, N.-W. Pu, S. Jeong, and J. Bokor, "Near-field propagation of terahertz pulses from a large-aperture antenna," Opt. Lett. 23, 213-215 (1998).

9. M. Lefrançois and S. F. Pereira, "Time evolution of the diffraction pattern of an ultrashort laser pulse," Opt. Express 11, 1114-1122 (2003).

10. M. Kempe, U. Stamm, B. Wilhelmi, and W. Rudolph, "Spatial and temporal transformation of femtosecond laser pulses by lenses and lens systems," J. Opt. Soc. Am. B 9, 1158-1165 (1992).

11. Z. Jiang, R. Jacquemin, and W. Eberhardt, "Time dependence of Fresnel diffraction of ultrashort laser pulses by a circular aperture," Appl. Opt. 36, 4358-4361 (1997).

12. M. Gu and X. S. Gan, "Fresnel diffraction by circular and serrated apertures illuminated with an ultrashort pulsed-laser beam," J. Opt. Soc. Am. A 13, 771-778 (1996).

13. D. P. Kelly, B. M. Hennelly, A. Grün, and K. Unterrainer, "Numerical sampling rules for paraxial regime pulse diffraction calculations," J. Opt. Soc. Am. A 25, 2299-2308 (2008).

14. Z. L. Horváth and Z. Bor, "Diffraction of short pulses with boundary diffraction wave theory," Phys. Rev. E 63, 026601 (2001).
15. Z. L. Horváth, J. Klebniczki, G. Kurdi, and A. P. Kovács, "Experimental investigation of the boundary wave pulse," Opt. Commun. 239, 243-250 (2004).

16. P. Saari, P. Bowlan, H. Valtna-Lukner, M. Lõhmus, P. Piksarv, and R. Trebino, "Basic diffraction phenomena in time domain," Opt. Express 18, 11083-11088 (2010).

17. M. Lõhmus, P. Bowlan, P. Piksarv, H. Valtna-Lukner, R. Trebino, and P. Saari, "Diffraction of ultrashort optical pulses from circularly symmetric binary phase gratings," Opt. Lett. 37, 1238-1240 (2012).

18. P. Piksarv, P. Bowlan, M. Lõhmus, H. Valtna-Lukner, R. Trebino, and P. Saari, "Diffraction of ultrashort Gaussian pulses within the framework of boundary diffraction wave theory," J. Opt. 14, 015701 (2012).

19. O. Mendoza-Yero, G. Mínguez-Vega, J. Lancis, M. FernándezAlonso, and V. Climent, "On-axis diffraction of an ultrashort light pulse by circularly symmetric hard apertures," Opt. Express 15, 4546-4556 (2007).

20. C. J. Zapata-Rodríguez, "Temporal effects in ultrashort pulsed beams focused by planar diffracting elements," J. Opt. Soc. Am. A 23, 2335-2341 (2006).

21. P. Saari and H. Sõnajalg, "Pulsed Bessel beams," Laser Phys. 7, 32-39 (1997).

22. M. A. Porras, R. Borghi, and M. Santarsiero, "Superluminality in Gaussian beams," Opt. Commun. 203, 183-189 (2002).

23. R. Borghi, F. Gori, and M. Santarsiero, "Optimization of Laguerre-Gauss truncated series,” Opt. Commun. 125, 197-203 (1996).

24. A. E. Siegman, Lasers (University Science, 1986).

25. J. A. Murphy and A. Egan, "Examples of Fresnel diffraction using Gaussian modes," Eur. J. Phys. 14, 121-127 (1993).

26. N. Trappe, J. A. Murphy, and S. Withington, "The Gaussian beam mode analysis of classical phase aberrations in diffraction-limited optical systems," Eur. J. Phys. 24, 403-412 (2003).

27. E. Cavanagh and B. D. Cook, "Gaussian-Laguerre description of ultrasonic fields-numerical example: circular piston," J. Acoust. Soc. Am. 67, 1136-1140 (1980).

28. Y. Liu and B. Lü, "Truncated Hermite-Gauss series expansion and its application," Optik 117, 437-442 (2006).

29. E. Cagniot, M. Fromager, and K. Ait-Ameur, "Modeling the propagation of apertured high-order Laguerre-Gaussian beams by a user-friendly version of the mode expansion method," J. Opt. Soc. Am. A 27, 484-491 (2010).

30. J. Durnin, J. J. Miceli, Jr., and J. H. Eberly, "Nondiffracting beams," Phys. Rev. Lett. 58, 1499-1501 (1987).

31. R. L. Phillips and L. C. Andrews, "Spot size and divergence for Laguerre Gaussian beams of any order," Appl. Opt. 22, 643-644 (1983).

32. I. S. Gradstehyn and I. M. Rhyzik, Table of Integrals, Series, and Products, 7th ed. (Academic, 2007), p. 811.

33. S. Feng and H. G. Winful, "Physical origin of the Gouy phase shift," Opt. Lett. 26, 485-487 (2001).

34. R. J. Mahon and J. A. Murphy, "Gaussian beam mode analysis of optical pulses," Proc. SPIE 8171, 81710H (2011).

35. Y. D. Liu and C. Gao, "Study on the time-varying and propagating characteristics of ultrashort pulse Laguerre-Gaussian beam," Opt. Express 18, 12104-12110 (2010).

36. E. Heyman and I. Beracha, "Complex multipole pulsed beams and Hermite pulsed beams: a novel expansion scheme for transient radiation from well-collimated apertures," J. Opt. Soc. Am. A 9, 1779-1973 (1992).

37. S. Feng and H. G. Winful, "Higher-order transverse modes of ultrashort isodiffracting pulses," Phys. Rev. E 63, 046602 (2001).

38. R. W. Ziolkowski and J. B. Judkins, "Propagation characteristics of ultrawide-bandwidth pulsed Gaussian beams," J. Opt. Soc. Am. A 9, 2021-2030 (1992).

39. A. B. Ruffin, J. V. Rudd, J. F. Whitaker, S. Feng, and H. G. Winful, "Direct observation of the Gouy phase shift with single cycle terahertz pulses,", Phys. Rev. Lett. 83, 3410-3413 (1999).

40. Z. L. Horváth and Z. Bor, "Reshaping of femtosecond pulses by the Gouy phase shift," Phys. Rev. E 60, 2337-2346 (1999).

41. S. Feng, H. G. Winful, and R. W. Hellwarth, "Gouy shift and temporal reshaping of focused single-cycle electromagnetic pulses," Opt. Lett. 23, 385-387 (1998). 
42. Z. Yang, Z. Yang, and S. Zhang, "Carrier-envelope phase of ultrashort pulsed Laguerre-Gaussian beam," Chin. Opt. Lett. 6, 189-191 (2008).

43. M. V. Vasnetsov and V. A. Pas'ko, "Group velocity of Gaussian beams," Ukr. J. Phys. 54, 50-52 (2009).

44. Q. Zou and B. Lü, "Anomalous spectral behaviour near phase singularities in diffraction of pulsed Laguerre-Gaussian beams," J. Opt. A 8, 531-536 (2006)

45. W. $\mathrm{Hu}$ and H. Guo, "Ultrashort pulsed Bessel beams and spatially induced group-velocity dispersion," J. Opt. Soc. Am. A 19, 49-53 (2002).

46. J. W. Goodman, Introduction to Fourier Optics, 2nd ed. (McGraw-Hill, 1996).

47. D. Deng, H. Guo, D. Han, M. Liu, and C. Li, "Effects of dispersion and longitudinal chromatic aberration on the focusing of isodiffracting pulsed Gaussian light beam," Phys. Lett. A 334 73-80 (2005).
48. Q. Zou and B. Lü, "Temporal and spectral properties of ultrashort pulsed Laguerre-Gaussian beams in dispersive media," Optik 118, 83-87 (2007).

49. R. W. Boyd, Nonlinear Optics, 3rd ed. (Academic, 2007), pp. 379-380.

50. J. J. Wen and M. A. Breazeale, "A diffraction beam field expressed as the superposition of Gaussian beams," J. Acoust. Soc. Am. 83, 1752-1756 (1988)

51. D. Ding and Y. Zhang, "Notes on the Gaussian beam expansion," J. Acoust. Soc. Am. 116, 1401-1405 (2004).

52. M. Zamboni-Rached, E. Recami, and M. Balma, "A simple and effective method for the analytic description of important optical beams, when truncated by finite apertures," Appl. Opt. 51, 3370-3379 (2012).

53. M. Zamboni-Rached, K. Z. Nóbrega, and C. A. Dartora, "Analytic description of Airy-type beams when truncated by finite apertures," Opt. Express 20, 19972-19977 (2012). 